\title{
Effect of bisphenol A on SOCS-3 and insulin signaling transduction in 3T3-L1 adipocytes
}

\author{
YUE-E DAI, WEI CHEN, HUMIN QI and QIAN-QI LIU \\ Department of Endocrinology, Nanjing Children's Hospital, Affiliated With Nanjing Medical University, \\ Nanjing, Jiangsu 210008, P.R. China
}

Received June 2, 2015; Accepted April 6, 2016

DOI: $10.3892 / \mathrm{mmr} .2016 .5224$

\begin{abstract}
The aim of the present study was to investigate whether environmental endocrine disrupting chemical, bisphenol A (BPA), affects secretion of suppressor of cytokine signaling 3 (SOCS-3) and insulin signaling transduction in 3T3-L1 adipocytes. 3T3-L1 adipocytes were treated for $0,2,6,12$ and $24 \mathrm{~h}$ with BPA at $80 \mu \mathrm{M}$ in serum-deprived medium. Reverse transcription-quantitative polymerase chain reaction and western blotting were performed to detect the mRNA expression levels of SOCS-3 and protein expression levels of SOCS-3, insulin receptor substrate 1 (IRS-1), phosphorylated (p)-IRS-1, Akt and p-Akt. The levels of p-IRS-1, Akt and p-Akt in cultures treated for $6 \mathrm{~h}$ with BPA were also analyzed by immunofluorescence. The SOCS-3 mRNA and protein expression levels were decreased in the 6,12 and $24 \mathrm{~h}$ groups. The levels of p-IRS-1 and p-Akt protein were markedly downregulated, while the level of IRS-1 and Akt protein remained unaltered among these groups, which was consistent with the results observed using immunofluorescence. BPA may inhibit insulin signal transduction and result in the occurrence of insulin resistance via promoting the expression of SOCS-3.
\end{abstract}

\section{Introduction}

The incidence of diabetes has notably increased in recent years, and it is estimated that 380 million patients will be diagnosed worldwide by 2025 (1). Insulin resistance (IR), a key feature of type 2 diabetes, is a state in which insulin has a reduced ability to mediate glucose homeostasis in its major target tissues, resulting in compensatory hyperinsulinemia. Previous studies have indicated that various signaling pathways, particularly insulin receptor substrate-1 (IRS-1)/phosphatidylinositol 3-kinase (PI3K)/Akt, are key in mediating the occurrence of IR $(2,3)$.

Correspondence to: Dr Qian-Qi Liu, Department of Endocrinology, Nanjing Children's Hospital, Affiliated With Nanjing Medical University, 72 Guangzhou Road, Nanjing, Jiangsu 210008, P.R. China E-mail: 18951769617@163.com

Key words: bisphenol A, SOCS-3, 3T3-L1 adipocytes
Insulin action involves a series of signaling transduction pathways, initiated by insulin binding to its receptor, which initiates receptor autophosphorylation and activation of the receptor tyrosine kinase, resulting in tyrosine phosphorylation of IRS-1, a substrate of the insulin receptor $(4,5)$. Subsequently, phosphorylation of IRS-1 leads to activation of PI3K and Akt and its downstream signaling molecules, all of which are important in the promotion of the synthesis of glycogen and regulation of glucose homeostasis (6). Thus, a state of IR often suggests the attenuation or failure of insulin signaling transduction by inhibiting the phosphorylation of key molecules.

Bisphenol A (BPA) is widely used as a plasticizer and stabilizer in the manufacture of consumer products, however, it is considered an endocrine disrupting chemical (EDC). Exposure to EDCs is proposed to be involved in the etiology of IR and associated metabolic disorders (7). Data from the U.S. National Health and Nutritional Examination Survey 2003-2008 reported a positive association between urinary BPA levels and an increased prevalence of diabetes mellitus independent of traditional diabetes risk factors (8), consistent with research findings in China (9). In addition, previous studies have elucidated the mechanisms by which BPA provoked IR by affecting glucose transport $(10,11)$, adiponectin secretion (12), adipocyte differentiation and lipid accumulation (13).

Furthermore, suppressor of cytokine signaling 3 (SOCS-3), a negative regulator of insulin signaling, is known to be associated with IR. A previous study demonstrated resistin induced IR in HepG2 cells via induction of SOCS-3 expression (14). It has also been previously reported that the expression levels of SOCS-3 were markedly increased in mice with IR induced by a high fat diet (15), which may contribute to increased serine phosphorylation of IRS-1. Insulin signaling transduction is impaired by inhibiting the activation of tyrosine phosphorylation of IRS-1 (16). However, to the best of our knowledge, no investigation into the effect of BPA on SOCS-3 has been conducted. The aim of the current study was to investigate whether BPA modulates SOCS-3 production and affects insulin signaling transduction in 3T3-L1 adipocytes.

\section{Materials and methods}

Reagents and antibodies. Dulbecco's modified Eagle's medium (DMEM) and fetal bovine serum (FBS) were purchased from Gibco (Thermo Fisher Scientific, Inc., Waltham, MA, USA). 
BPA, dexamethasone, insulin and 3-isobuty-1-methylxanthine (IBMX) were obtained from Sigma-Aldrich (St. Louis, MO, USA). Rabbit polyclonal anti-SOCS-3 antibody (cat. no. 2923), rabbit monoclonal anti-IRS-1 antibody (cat. no. 2390), rabbit polyclonal anti-Akt antibody (cat. no. 9272) and rabbit polyclonal anti-phosphorylated (p)-Akt (Ser473) antibody (cat. no. 9271) were purchased from Cell Signaling Technology, Inc. (Danvers, MA, USA; dilution, 1:1,000 for western blotting). Goat polyclonal anti-p-IRS-1 (Tyr 632) antibody (cat. no. sc-17196) was purchased from Santa Cruz Biotechnology, Inc. (Dallas, TX, USA; dilution, 1:200). Mouse monoclonal antibodies against $\beta$-actin (cat.no. ab8226; dilution, 1:1000), horseradish peroxidase (HRP)-conjugated goat anti-rabbit immunoglobulin $\mathrm{G}$ ( $\mathrm{IgG})$ (cat. no. ab6721; dilution 1:200), horseradish peroxidase goat anti-mouse IgG (cat. no. ab6789; dilution, 1:200), horseradish peroxidase-conjugated donkey anti-goat IgG (cat. no. ab6885; dilution, 1:100), fluorescein isothiocyanate (FITC)-conjugated polyclonal donkey anti-goat IgG (cat. no. ab6881; dilution, 1:200), FITC-conjugated polyclonal goat anti-rabbit IgG (cat. no. ab6717; dilution, 1:100) and cyanine 3 (Cy3)-conjugated goat anti-rabbit IgG (cat. no. 97075; dilution, 1:100) secondary antibodies were purchased from Abcam (Cambridge, MA, USA).

Cell culture and treatment. The 3T3-L1 mouse preadipocyte cell line was obtained from the American Type Culture Collection (Manassas, VA, USA), and the cells were cultured and differentiated into adipocytes as described previously (17). Briefly, the cells were grown in DMEM containing 10\% FBS, $100 \mathrm{U} / \mathrm{ml}$ penicillin (Beyotime Institute of Biotechnology, Haimen, China) and $100 \mathrm{mg} / \mathrm{ml}$ streptomycin (Beyotime Institute of Biotechnology) at $37^{\circ} \mathrm{C}$ in a humidified atmosphere of $5 \% \mathrm{CO}_{2}$. The cells were exposed to standard differentiation inducers $48 \mathrm{~h}$ after confluency was reached. The inducer used was DMEM containing $0.5 \mathrm{mM}$ IBMX, $1 \mu \mathrm{M}$ dexamethasone and $10 \mu \mathrm{g} / \mathrm{ml}$ insulin for $48 \mathrm{~h}$ (from day 0 to 2 ). The medium was then changed and supplemented with $10 \mu \mathrm{g} / \mathrm{ml}$ insulin only for the following $48 \mathrm{~h}$ (from day 2 to 4 ). Thereafter, the medium was replaced by growth medium and changed every 2 days. At 10 days after the induction of differentiation, $>80 \%$ of cells exhibited typical morphology and biochemical properties of adipocytes. Following overnight incubation in serum-free DMEM, 3T3-L1 adipocytes were treated with $80 \mu \mathrm{M}$ BPA diluted in DMEM for 0, 2, 6, 12 and 24 h respectively.

$R N A$ preparation and reverse transcription-quantitative polymerase chain reaction $(R T-q P C R)$. Total RNA from 3T3-L1 adipocytes treated with BPA was extracted using TRIzol (Invitrogen; Thermo Fisher Scientific, Inc.) according to the manufacturer's protocols and quantified spectrophotometrically by measuring absorbance at wavelengths 260 and $280 \mathrm{~nm}$ on a BioPhotometer spectrophotometer (Eppendorf, Hamburg, Germany). RT was conducted using Transcriptor First Strand cDNA Synthesis kit (Roche Diagnostics, Basel, Switzerland) from $1 \mu \mathrm{g}$ RNA as described by the manufacturer's protocols. The temperature protocol for the reaction was $25^{\circ} \mathrm{C}$ for $10 \mathrm{~min}, 55^{\circ} \mathrm{C}$ for $30 \mathrm{~min}$ and $85^{\circ} \mathrm{C}$ for $5 \mathrm{~min}$. qPCR was performed to determine the relative mRNA expression levels of SOCS-3. $\beta$-actin served as an internal control for normalization. Specific mRNAs were amplified in FastStart Universal SYBR Green Master mix (Roche Diagnostics) using the ABI Prism 7500 (Applied Biosystems; Thermo Fisher Scientific, Inc.) sequence detector with the following thermocycling conditions: $50^{\circ} \mathrm{C}$ for $2 \mathrm{~min} ; 95^{\circ} \mathrm{C}$ for $10 \mathrm{~min}$; 40 cycles of $95^{\circ} \mathrm{C}$ for $15 \mathrm{sec}$ and $60^{\circ} \mathrm{C}$ for $1 \mathrm{~min}$. The primers (Invitrogen; Thermo Fisher Scientific, Inc.) were as follows: Forward, 5'-ATGGTCACCCACAGCAAGTTT-3' and reverse, 5'-TCCAGTAGAATCCGCTCTCCT-3' for SOCS-3; and forward, 5'-CTACAATGAGCTGCGTGTGG-3' and reverse, 5'-AAGGAAGGCTGGAAGAGTGC-3' for $\beta$-actin. Three data points were used and the experiment was replicated three times and the data was analyzed using the $2^{-\Delta \Delta \mathrm{Cq}}$ method (18).

Protein extraction and western blot analysis. At 0,2, 6, 12 and $24 \mathrm{~h}$, the cells treated with BPA were washed twice with ice-cold phosphate-buffered saline (PBS; Thermo Fisher Scientific, Inc.) and lysed in radioimmunoprecipitation assay buffer (Beyotime Institute of Biotechnology) supplemented with $1 \%$ protease inhibitor (GeneChem Co., Ltd., Shanghai, China) and $1 \%$ phosphatase inhibitor (GeneChem Co., Ltd.). Total and phosphorylated proteins were extracted and concentration was determined using the BCA Protein assay kit (Beyotime Institute of Biotechnology). Following 10\% sodium dodecyl sulfate-polyacrylamide gel electrophoresis for $30 \mathrm{~min}$ at $80 \mathrm{~V}$ followed by $120 \mathrm{~V}$, the protein bands ( $20 \mu \mathrm{g} /$ lane) were electrophoretically transferred onto polyvinyl difluoride membranes (GE Healthcare Life Sciences, Chalfont, UK). The blots were blocked for $2 \mathrm{~h}$ at room temperature in Tris-buffered saline (Beyotime Institute of Biotechnology), with 0.1\% Tween 20 (Sigma-Aldrich; TBS-T) and $5 \%$ non-fat dried milk and subsequently incubated overnight at $4^{\circ} \mathrm{C}$ with the primary antibodies against SOCS3, IRS-1, p-IRS-1, Akt, p-Akt and $\beta$-actin. Following washing in TBS-T buffer, the membranes were incubated with HRP-conjugated secondary antibodies at room temperature for $1 \mathrm{~h}$. Following washing in TBS-T, antigen-antibody complexes were detected using Amersham ECL Western Blotting Detection reagent (GE Healthcare Life Sciences) and visualized with the ChemiDoc XRS imaging system (Bio-Rad Laboratories, Inc., Hercules, CA, USA) and quantified using the Quantity One image software (version 4.31; Bio-Rad Laboratories, Inc.).

Immunocytochemistry. After $6 \mathrm{~h}$ treatment with BPA, 3T3-L1 adipocytes were fixed for $30 \mathrm{~min}$ with $4 \%$ paraformaldehyde (Sigma-Aldrich) at room temperature, and then rinsed three times for $5 \mathrm{~min}$ with PBS. The cells were permeabilized for $10 \mathrm{~min}$ in $0.1 \%$ Triton $\mathrm{X}-100$ (Sigma-Aldrich), then again rinsed twice for $5 \mathrm{~min}$ in PBS, and blocked for $1 \mathrm{~h}$ in PBS with $5 \%$ bovine serum albumin (Sigma-Aldrich) at room temperature. Antibodies against p-IRS-1 (dilution, 1:200), Akt (dilution, 1:200) and anti-p-Akt (dilution, 1:200) were incubated with the cells at $4^{\circ} \mathrm{C}$ overnight. The cells were then incubated with FITC-conjugated goat anti-rabbit IgG (1:500) or Cy3-conjugated goat anti-rabbit IgG (1:500) at room temperature for $2 \mathrm{~h}$, followed by washing in PBS. The cells were stained with DAPI (Sigma-Aldrich) for $3 \mathrm{~min}$ and images were captured using a Nikon Eclipse Ti-S fluorescent inverted microscope (Nikon Corporation, Tokyo, Japan) at magnification $\mathrm{x} 200$.

Statistical analysis. All data are expressed as the mean \pm standard deviation. Differences among 3-5 independent groups 
were statistically evaluated using one-way ANOVA, while significant differences between 2 independent groups were analyzed using Student's t-test. Statistical analysis was conducted using SPSS 13.0 (SPSS, Inc., Chicago, IL, USA) and $\mathrm{P}<0.05$ was considered to indicate a statistically significant difference.

\section{Results}

BPA increases SOCS-3 expression levels in 3T3-L1 adipocytes. The mRNA expression levels of SOCS-3 were analyzed using RT-qPCR and the results indicated that BPA treatment at $80 \mu \mathrm{M}$ significantly increased mRNA expression levels in time-dependent manners $(\mathrm{P}<0.01$ at $2-12 \mathrm{~h}$ and $\mathrm{P}<0.05$ at $24 \mathrm{~h}$; Fig. 1). In order to further investigate the protein expression of SOCS-3, western blotting was performed and the result is presented in Fig. 2. RT-qPCR and western blot analysis indicated that BPA significantly increased SOCS-3 mRNA and protein expression levels after 6 and $12 \mathrm{~h}$ of treatment compared to $0 \mathrm{~h}(\mathrm{P}<0.01)$. In addition, the expression levels of SOCS-3 mRNA and protein were overexpressed after $24 \mathrm{~h}$ of treatment with BPA $(\mathrm{P}<0.05)$.

BPA alters protein expression levels of insulin signaling molecules in 3T3-L1 adipocytes. 3T3-L1 adipocytes were treated with $80 \mu \mathrm{M}$ BPA for 0,2, 6, 12 and $24 \mathrm{~h}$ and the expression levels of IRS-1, p-IRS-1, Akt and p-Akt were analyzed by western blotting. As presented in Fig. 3, there were no significant differences in the protein expression levels of IRS-1 among these groups. However, BPA decreased the expression levels of p-IRS-1 at $6 \mathrm{~h}$ of treatment compared with $0 \mathrm{~h}$ $(\mathrm{P}<0.01)$. Similar effects were also observed in the level of Akt (Fig. 4). However, a significant decrease in expression levels of p-Akt was observed following treatment with BPA for 2 to $24 \mathrm{~h}(\mathrm{P}<0.01$; Fig. 4). These results suggest that BPA markedly decreased the expression levels of insulin signaling molecules in 3T3-L1 adipocytes.

BPA decreased expression levels of $p$-IRS-1 and p-Akt. To further elucidate the effect of BPA, immunocytochemistry was conducted to investigate the expression levels of the insulin signaling molecules. Consistent with the results from the western blotting, p-IRS-1 (Fig. 5) and p-Akt (Fig. 6) expression levels were markedly decreased in BPA-treated cells compared with control cells, while expression levels of Akt did not exhibit a marked change (Fig. 6).

\section{Discussion}

Adipose tissue is important in insulin sensitivity and basal metabolic rate. Thus, 3T3-L1 adipocytes were selected to investigate the effects of BPA on SOCS-3 and insulin signaling transduction. In the current study, it was observed that BPA significantly increased SOCS-3 secretion in 3T3-L1 adipocytes $(\mathrm{P}<0.01)$ and decreased the expression of key molecules involved in the IRS-1/PI3K/Akt signaling pathway.

BPA, in addition to other environmental estrogens, has become a public health concern due to deleterious effects on energy balance and glucose homeostasis (19). The present study indicates that BPA exposure impairs insulin signaling in peripheral tissues and may be a risk factor for the development of type 2 diabetes (20). During the early stages of life, BPA exposure may impair pancreatic development and result in adults susceptible to diabetes (21). In epidemiological studies in humans, $>93 \%$ of US adults have detectable BPA levels in urine, higher levels are particularly observed in the population with diabetes, hypertension and obesity (22). In animal models, BPA exposure in pregnant rats increased their offspring's body weight, and the levels of fasting blood glucose and serum insulin, which may predispose them to IR (23). Data from a previous study demonstrated that BPA exhibited estrogen-like activities via binding to estrogen receptors (ERs), non-classical membrane ERs, G-protein-coupled receptor 30 and estrogen-related receptors (24). There were, however, few studies that had investigated the effect of BPA on insulin signal transduction, thus, the present study aimed to investigate the association between BPA and the IRS-1/PI3K/Akt signaling pathway.

As previously described, IR may be induced by the inhibition of insulin signaling transduction. In the current study, the results of the western blotting indicated that BPA significantly decreased the expression levels of p-IRS-1 and p-Akt $(\mathrm{P}<0.01)$, which are key in insulin-stimulated glucose transport (25). The decrease in protein expression levels of p-IRS-1 and p-Akt were further shown by immunocytochemistry. In vitro, the cellular uptake of glucose into the cells by glucose transporters requires insulin and receptor-mediated tyrosine phosphorylation of IRS-1 (26), which is key in insulin signal transduction and affects insulin signaling by regulating protein presentation, post-translational modification and subcellular localization of proteins, particularly in phosphorylation/dephosphorylation of post-translational modification (27). IRS-1 is closely associated with PI3K activation, which is responsible for activation of the Akt signaling cascade (28). It is generally accepted that impaired tyrosine phosphorylation of IRS-1 is responsible for reduced insulin signaling and impaired downstream PI3K/Akt signal transduction (29). The downregulated phosphorylation of Akt resulting from attenuated tyrosine phosphorylation of IRS-1 may impair GLUT4 translocation and glucose uptake. A previous study has indicated that insulin-stimulated Akt phosphorylation was suppressed in skeletal muscle and livers of BPA-treated pregnant mice, these mice then suffered from metabolic disorders associated with glucose homeostasis (30). The present study suggests that $80 \mu \mathrm{M}$ BPA may inhibit the IRS-1/PI3K/Akt signaling pathway, which results in IR.

To further investigate the underlying mechanisms of BPA induced impairment of insulin signaling transduction, the mRNA and protein expression levels of SOCS-3 were investigated by RT-qPCR and western blotting. The results demonstrated that BPA markedly increased SOCS-3 mRNA and protein expression levels in a time-dependent manner. In addition, it was observed that tyrosine phosphorylation of IRS-1 and serine phosphorylation of Akt was decreased as demonstrated by a decrease in the expression levels of these proteins following the treatment with BPA. This was consistent with the increased expression levels of SOCS-3 at the same time points.

SOCS-3 is one member of the SOCS protein family, which is overexpressed in insulin-sensitive tissues from patients 


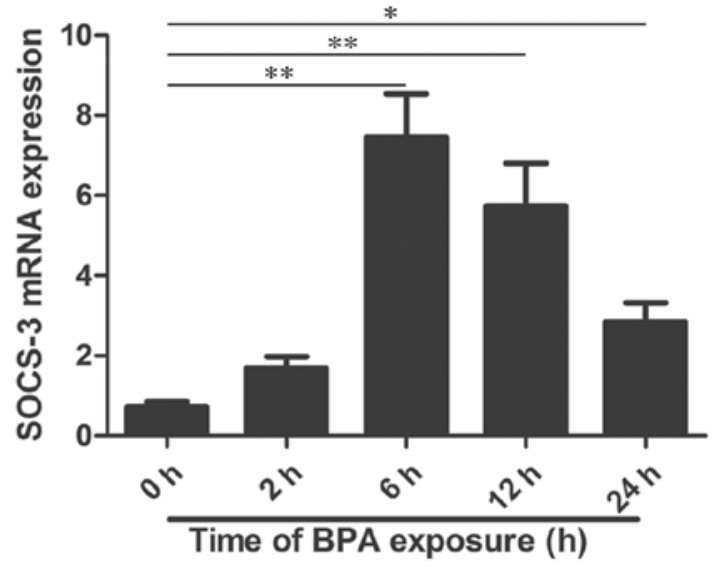

Figure 1. mRNA expression levels of SOCS-3. The mRNA expression levels of SOCS-3 were detected by reverse transcription-quantitative polymerase chain reaction at $0,2,6,12$ and $24 \mathrm{~h}$ after BPA treatment. ${ }^{*} \mathrm{P}<0.05,{ }^{* *} \mathrm{P}<0.01$. BPA, bisphenol A; SOCS-3, suppressor of cytokine signaling 3.

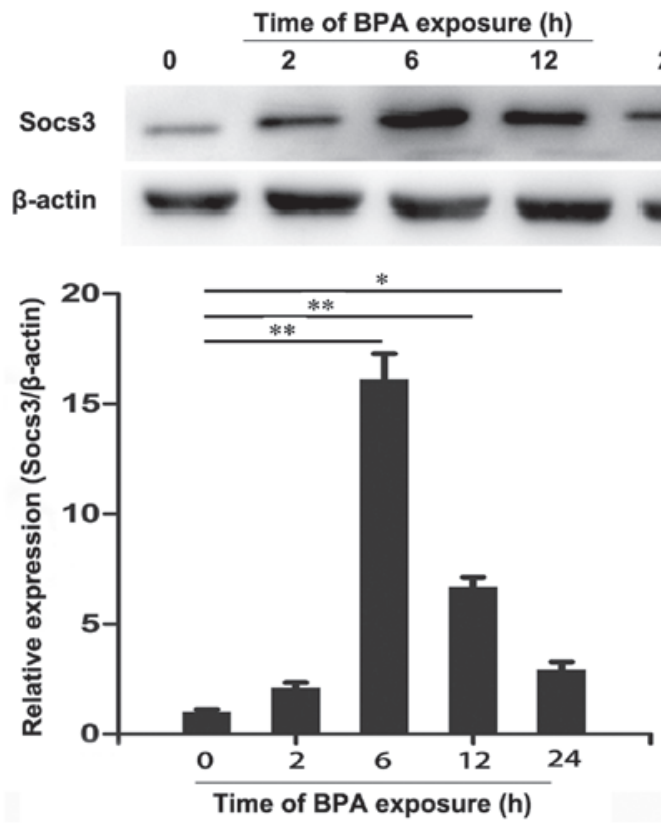

Figure 2. Western blot analysis of SOCS-3 expression. The expression levels of SOCS-3 and $\beta$-actin were detected by western blot at $0,2,6,12$ and $24 \mathrm{~h}$ after BPA treatment. ${ }^{*} \mathrm{P}<0.05,{ }^{* *} \mathrm{P}<0.01$. BPA, bisphenol A; SOCS-3, suppressor of cytokine signaling 3 .

with type 2 diabetes and IR and animal models of the conditions $(31,32)$. Previous studies have demonstrated SOCS-3 binds via the $\mathrm{SH} 2$ domain to tyrosine phosphorylation sites on cytokine receptors to inhibit inflammatory signal transduction (33). In the skeletal muscle of obese Zucker rats, SOCS-3 protein concentration and co-localization of SOCS-3 with IRS-1 is notably increased, while tyrosine phosphorylation of IRS-1 was decreased and serine phosphorylation of IRS-1 was increased (34). Furthermore, mice with muscle-specific deletion of SOCS-3 were protected against the development of hyperinsulinemia and IR due to enhanced skeletal muscle IRS-1 and Akt phosphorylation (16). Similarly, genetic deletion of SOCS-3 from mouse liver also results in enhanced insulin signaling due to

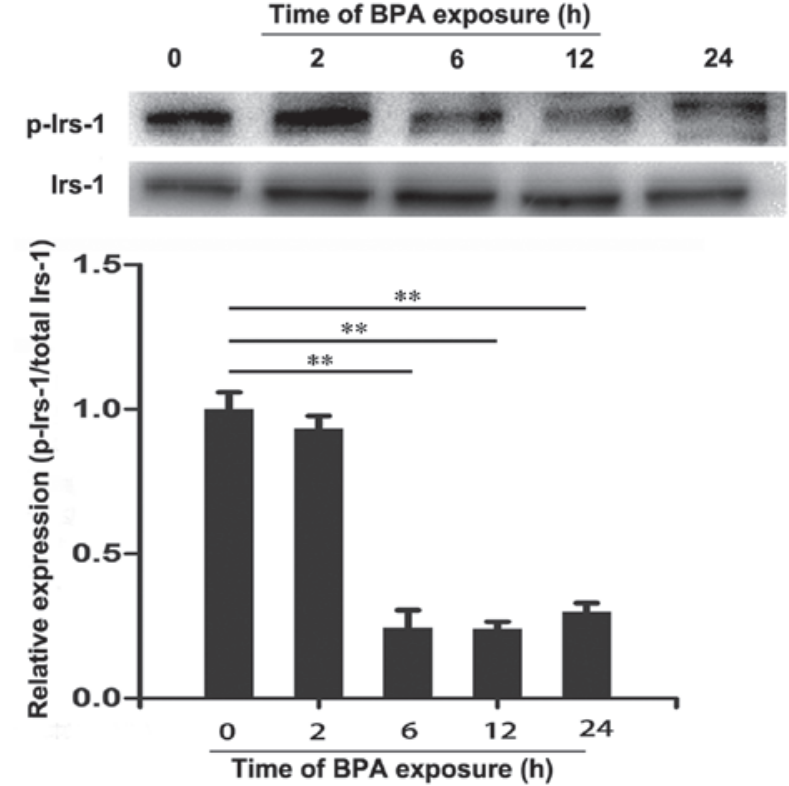

Figure 3. Western blot analysis of p-IRS-1 and IRS-1. The expression levels of p-IRS-1 and IRS-1 were detected by western blotting at 0,2, 6, 12 and $24 \mathrm{~h}$ after BPA treatment. ${ }^{* *} \mathrm{P}<0.01$. BPA, bisphenol A; IRS-1, insulin receptor substrate $1 ;$ p, phosphorylated.

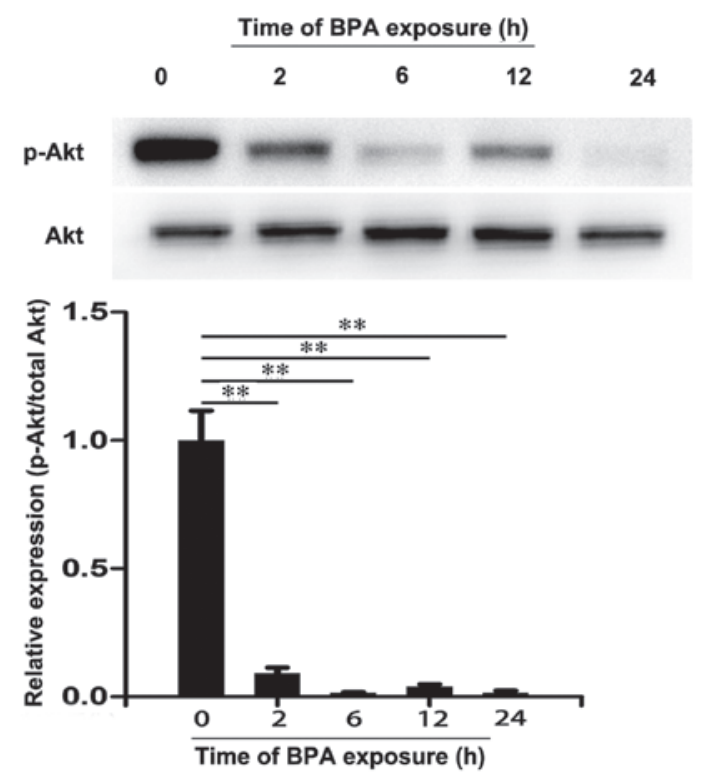

Figure 4. Western blot analysis of p-Akt and Akt. The expressions of p-AKT and AKT were detected by western blotting at $0,2,6,12$ and $24 \mathrm{~h}$ after BPA treatment. ${ }^{* *} \mathrm{P}<0.01$. BPA, bisphenol A; p, phosphorylated.

increased IRS-1 phosphorylation (35). These studies suggest SOCS-3 interferes with insulin signaling and results in IR by inhibiting tyrosine phosphorylation of IRS-1.

In conclusion, BPA significantly increases mRNA and protein expression levels of SOCS-3 and decreases the phosphorylation of IRS-1 and Akt. Based on these results, the present study hypothesizes that BPA may inhibit insulin signal transmission and lead to the development of IR via promoting the expression of SOCS-3 and preventing tyrosine phosphorylation of IRS- 1 . The present study provides a novel insight into the mechanism by which BPA induces IR. 

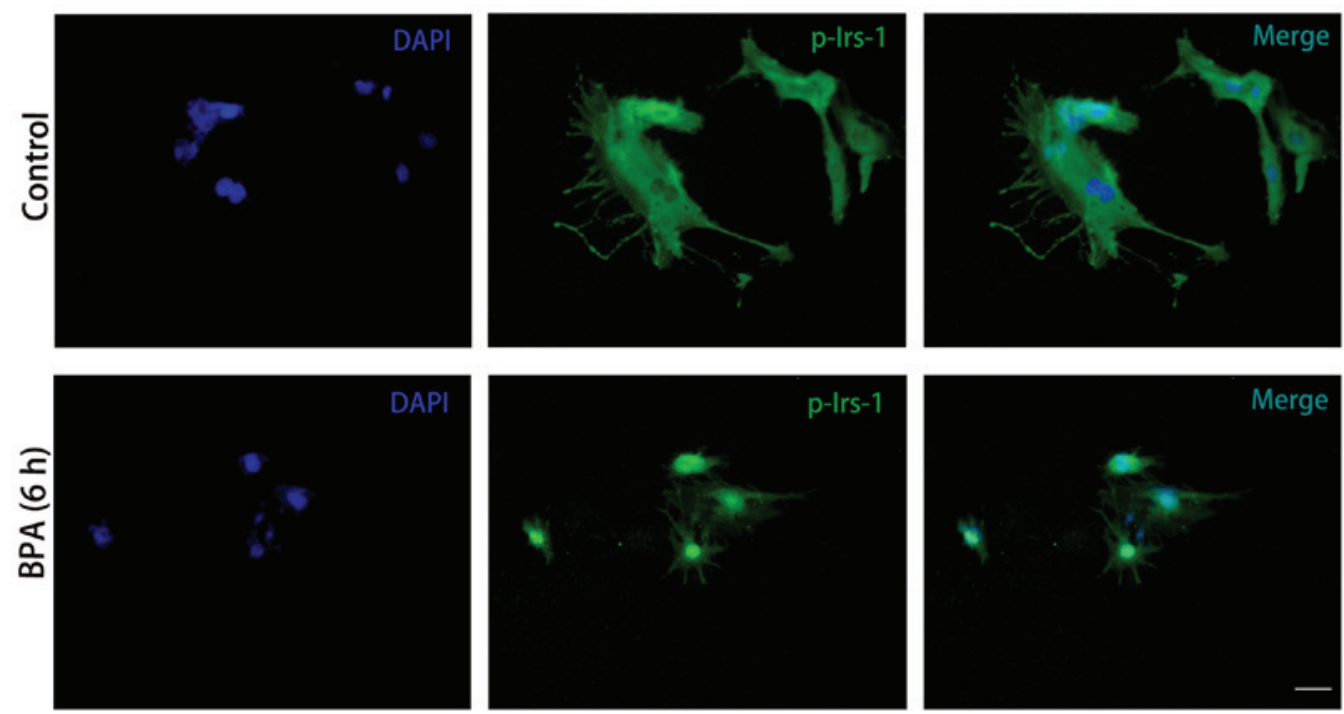

Figure 5. Immunocytochemistry of p-IRS-1. Expression levels of p-IRS-1 were markedly lower in BPA-treated cells after $6 \mathrm{~h}$. Bar $=50 \mu \mathrm{m}$. Magnification, x200. BPA, bisphenol A; IRS-1, insulin receptor substrate 1 .
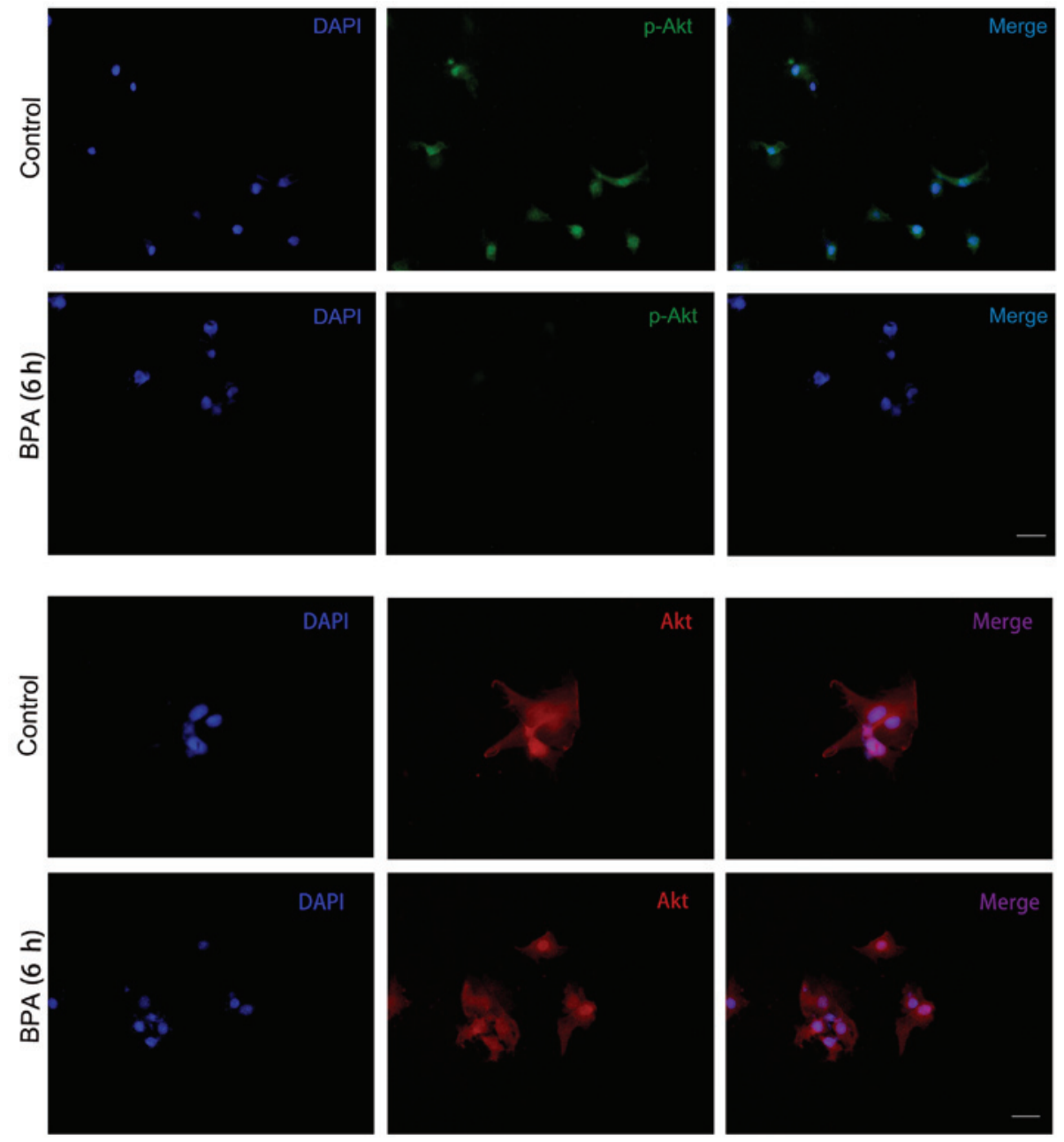

Figure 6. Immunocytochemistry of p-Akt and Akt. Expression levels of p-Akt were markedly lower in BPA-treated cells after $6 \mathrm{~h}$, while Akt expression exhibited no marked change. Bar $=50 \mu \mathrm{m}$. Magnification, $\mathrm{x} 200$. BPA, bisphenol A; p, phosphorylated.

\section{Acknowledgements}

The present study was supported by grants from the
National Key Basic Research Program of China (grant no. 2013CB530604), the National Natural Science Foundation of China (grant nos. 30973231 and 81270928), the Talent 
Foundation of Jiangsu Province, China (grant no. QRX11051), and the Science and Technology Development Foundation of Nanjing Medical University (grant no. 2014NLMU144).

\section{References}

1. Zhu S, Sun F, Li W, Cao Y, Wang C, Wang Y, Liang D, Zhang R, Zhang S, Wang H and Cao F: Apelin stimulates glucose uptake through the PI3K/Akt pathway and improves insulin resistance in 3T3-L1 adipocytes. Mol Cell Biochem 353: 305-313, 2011.

2. Avogaro A, de Kreutzenberg SV and Fadini GP: Oxidative stress and vascular disease in diabetes: Is the dichotomization of insulin signaling still valid? Free Radic Biol Med 44: 1209-1215, 2008.

3. Galadari S, Rahman A, Pallichankandy S, Galadari A and Thayyullathil F: Role of ceramide in diabetes mellitus: Evidence and mechanisms. Lipids Health Dis 12: 98, 2013.

4. Esposito DL, Li Y, Cama A and Quon MJ: Tyr(612) and $\operatorname{Tyr}(632)$ in human insulin receptor substrate-1 are important for full activation of insulin-stimulated phosphatidylinositol 3 -kinase activity and translocation of GLUT4 in adipose cells. Endocrinology 142: 2833-2840, 2001.

5. Tsai CW, Liu KL, Lin YR and Kuo WC: The mechanisms of carnosic acid attenuates tumor necrosis factor- $\alpha$-mediated inflammation and insulin resistance in 3T3-L1 adipocytes. Mol Nutr Food Res 58: 654-664, 2014.

6. Choi K and Kim YB: Molecular mechanism of insulin resistance in obesity and type 2 diabetes. Korean J Intern Med 25: 119-129, 2010

7. Padmanabhan V, Sarma HN, Savabieasfahani M, Steckler TL and Veiga-Lopez A: Developmental reprogramming of reproductive and metabolic dysfunction in sheep: Native steroids vs. environmental steroid receptor modulators. Int J Androl 33 : 394-404, 2010

8. Shankar A and Teppala S: Relationship between urinary bisphenol A levels and diabetes mellitus. J Clin Endocrinol Metab 96: 3822-3826, 2011

9. Wang T, Li M, Chen B, Xu M, Xu Y, Huang Y, Lu J, Chen Y, Wang W, Li X, et al: Urinary bisphenol A (BPA) concentration associates with obesity and insulin resistance. J Clin Endocrinol Metab 97: E223-E227, 2012.

10. Ropero AB, Alonso-Magdalena P, Garcia-Garcia E, Ripoll C, Fuentes $\mathrm{E}$ and Nadal A: Bisphenol-A disruption of the endocrine pancreas and blood glucose homeostasis. Int J Androl 31: 194-200, 2008

11. Sakurai K, Kawazuma M, Adachi T, Harigaya T, Saito Y, Hashimoto $\mathrm{N}$ and Mori C: Bisphenol A affects glucose transport in mouse 3T3-F442A adipocytes. Br J Pharmacol 141: 209-214, 2004

12. Kidani T, Kamei S, Miyawaki J, Aizawa J, Sakayama K and Masuno H: Bisphenol A downregulates Akt signaling and inhibits adiponectin production and secretion in 3T3-L1 adipocytes. J Atheroscler Thromb 17: 834-843, 2010.

13. Sargis RM, Johnson DN, Choudhury RA and Brady MJ: Environmental endocrine disruptors promote adipogenesis in the 3T3-L1 cell line through glucocorticoid receptor activation. Obesity (Silver Spring) 18: 1283-1288, 2010.

14. Luo Z, Zhang Y, Li F, He J, Ding H, Yan L and Cheng H: Resistin induces insulin resistance by both AMPK-dependent and AMPK-independent mechanisms in HepG2 cells. Endocrine 36: 60-69, 2009 .

15. Ghanim H, Abuaysheh S, Sia CL, Korzeniewski K, Chaudhuri A, Fernandez-Real JM and Dandona P: Increase in plasma endotoxin concentrations and the expression of toll-like receptors and suppressor of cytokine signaling-3 in mononuclear cells after a high-fat, high-carbohydrate meal: Implications for insulin resistance. Diabetes Care 32: 2281-2287, 2009.

16. Jorgensen SB, O'Neill HM, Sylow L, Honeyman J, Hewitt KA, Palanivel R, Fullerton MD, Öberg L, Balendran A, Galic S, et al: Deletion of skeletal muscle SOCS3 prevents insulin resistance in obesity. Diabetes 62: 56-64, 2013.
17. Xie XY, Kong PR, Wu JF, Li Y and Li YX: Curcumin attenuates lipolysis stimulated by tumor necrosis factor- $\alpha$ or isoproterenol in 3T3-L1 adipocytes. Phytomedicine 20: 3-8, 2012.

18. Livak KJ and Schmittgen TD: Analysis of relative gene expression data using real-time quantitative PCR and the 2(-Delta Delta C(T)) method. Methods 25: 402-408, 2001.

19. Wei J, Lin Y, Li Y, Ying C, Chen J, Song L, Zhou Z, Lv Z, Xia W, Chen X and Xu S: Perinatal exposure to bisphenol A at reference dose predisposes offspring to metabolic syndrome in adult rats on a high-fat diet. Endocrinology 152: 3049-3061, 2011.

20. Batista TM, Alonso-Magdalena P, Vieira E, Amaral ME, Cederroth CR, Nef S, Quesada I, Carneiro EM and Nadal A: Short-term treatment with bisphenol-A leads to metabolic abnormalities in adult male mice. PloS One 7: e33814, 2012.

21. Liu J, Yu P, Qian W, Li Y, Zhao J, Huan F, Wang J and Xiao H: Perinatal bisphenol A exposure and adult glucose homeostasis: Identifying critical windows of exposure. PloS One 8: e64143, 2013.

22. Shankar A and Teppala S: Urinary bisphenol A and hypertension in a multiethnic sample of US adults. J Environ Public Health 2012: 481641, 2012.

23. Liu L, Ma C, Wen Z, Zhang L, Zhang Z and Jia L: Effect of bisphenol A exposure during early development on body weight and glucose metabolism of female filial rats. Wei Sheng Yan Jiu 41: 543-545, 2012 (In Chinese).

24. Ben-Jonathan N, Hugo ER and Brandebourg TD: Effects of bisphenol $\mathrm{A}$ on adipokine release from human adipose tissue: Implications for the metabolic syndrome. Mol Cell Endocrinol 304: 49-54, 2009.

25. Jayashree S, Indumathi D, Akilavalli N, Sathish S, Selvaraj J and Balasubramanian K: Effect of Bisphenol-A on insulin signal transduction and glucose oxidation in liver of adult male albino rat. Environ Toxicol Pharmacol 35: 300-310, 2013

26. D'Cruz SC, Jubendradass R, Jayakanthan M, Rani SJ and Mathur PP: Bisphenol A impairs insulin signaling and glucose homeostasis and decreases steroidogenesis in rat testis: An in vivo and in silico study. Food Chem Toxicol 50: 1124-1133, 2012.

27. Copps KD and White MF: Regulation of insulin sensitivity by serine/threonine phosphorylation of insulin receptor substrate proteins IRS1 and IRS2. Diabetologia 55: 2565-2582, 2012.

28. Guo S: Insulin signaling, resistance and the metabolic syndrome: Insights from mouse models into disease mechanisms. J Endocrinol 220: T1-T23, 2014.

29. Schenk S, Saberi M and Olefsky JM: Insulin sensitivity: Modulation by nutrients and inflammation. J Clin Invest 118: 2992-3002, 2008.

30. Alonso-Magdalena P, Vieira E, Soriano S, Menes L, Burks D, Quesada I and Nadal A: Bisphenol A exposure during pregnancy disrupts glucose homeostasis in mothers and adult male offspring. Environ Health Perspect 118: 1243-1250, 2010.

31. Lebrun P and Van Obberghen E: SOCS proteins causing trouble in insulin action. Acta Physiol (Oxf) 192: 29-36, 2008.

32. Zheng YY, Wang LF, Fan XH, Wu CH, Huo N, Lu HY, Xu XY and Wei L: Association of suppressor of cytokine signalling 3 polymorphisms with insulin resistance in patients with chronic hepatitis C. J Viral Hepat 20: 273-280, 2013.

33. Suchy D, Łabuzek K, Machnik G, Kozłowski M and Okopień B: SOCS and diabetes-ups and downs of a turbulent relationship. Cell Biochem Funct 31: 181-195, 2013.

34. Zolotnik IA, Figueroa TY and Yaspelkis BB III: Insulin receptor and IRS- 1 co-immunoprecipitation with SOCS-3 and IKK $\alpha / \beta$ phosphorylation are increased in obese Zucker rat skeletal muscle. Life Sci 91: 816-822, 2012.

35. Sachithanandan N, Fam BC, Fynch S, Dzamko N, Watt MJ, Wormald S, Honeyman J, Galic S, Proietto J, Andrikopoulos S, et al: Liver-specific suppressor of cytokine signaling-3 deletion in mice enhances hepatic insulin sensitivity and lipogenesis resulting in fatty liver and obesity. Hepatology 52: 1632-1642, 2010. 